\title{
Ground moving target detection using LTE-based passive radar
}

\begin{abstract}
This paper examines the feasibility of 4G (LTE)-based passive radar for detecting ground moving targets. Specifically, the focus of this paper is to describe the proposed LTE-based passive radar system and to conduct an experiment using a real LTE eNB transmitter as an illumination source. Seven scenarios were carried out to investigate the detection performance of the proposed system on moving targets. Each scenario had different types of ground targets moving with a variety of speeds and directions. The experimental results showed that the LTE-based passive radar system has the capability to detect typical ground targets/objects like cars, motorbikes and humans moving with different speeds, trajectories and ranges. The positive results opened up a new frontier for passive radar systems to be used in many potential applications, including border protection, microwave fences, monitor of buildings and others.
\end{abstract}

Keyword: Detection; LTE; Passive radar 